When the patient was old or enfeebled by previous use of mercury, \&c., better diet was allowed according to circumstances. In cases of bubo the quantity of food was increased, after opening the tumor; and when the healing process commenced animal food was first permitted.

A mild purgative of saline mixture was administered every two or three days, aided by a few grains of jalap in cases where the bowels were constipated.

Treatment of Complications. - Phymosis, occurring as a consequence of inflammation in a moderate degree, was treated antiphlogistically. In most cases of this kind the phymosis was cured by the use of warm lotions and the tepid bath. When the inflammatory symptoms, however, were more severe, and seemed to threaten mortification, the main reliance was placed on cold applications; the use of leeches was of little service. When a portion of the prepuce had already sloughed, the separation of the mortified parts was left to nature, and the healing promoted by a gradual change from cold to tepid local applications.

Paraphymosis of short duration, and accompanied by slight inflammation, yielded to pressure with the fingers; but in more severe cases the greatest benefit was obtained from the application of ice-cold lotions.

As to the use of the knife in any cases of paraphymosis, I regard it to be both superfluous and useless. Superfluous, because the stricture has been overcome by the use of cold in many cases where division was thought necessary ; and useless, because the operation is inapplicable to such cases where the stricture is produced by the inner layer of the prepuce embracing the edge of the corona glandis.

Inflammation of the lymphatic glands is always an unpleasant complication. As a primary symptom without previous chancre I saw only one example; this occurred fourteen days after unclean connection, and no ulcer or trace of ulcer could be detected.

The treatment of bubo consisted in rest, the use of fomentations, purgatives, and compression. According to my experience the most efficacious means are rest and the horizontal position, next to fomentations and purgatives. Warm emollient fomentations were preferred to cold. As compression was used in conjunction with many other means, it is not easy to estimate its real value.

Suppurating bubos were opened by incision in the long diameter, and then treated as simple abscesses, being carefully protected from the air. When granulation commenced, this process was aided by a lotion, or touching the part with lunar caustic, or an ointment containing fifteen grains of red precipitate, and two drachms of tincture of opium to two ounces of lard. When the integuments were extensively detached, thinned, and livid, the Vienna caustic was employed to open the bubos. When the tumor is opened in proper time it generally heals within twentyone days; if the incision be made before fluctuation is evident, the edges of the wound are apt to become everted and callous, and it is subsequently necessary to pare its edges with the knife or cut them down with caustic; on the other hand, if the opening be delayed too long, we have to apprehend sloughing or burrowing of the pus under the integuments.

Indurated bubo, arising from external causes during gonorrhœa, required the persevering use of iodine or some of its preparations. I saw only one example of bubo resulting from condyloma. A patient, twentythree years of age, had several pedunculated condylomata on the glans; these were removed by the knife and by ligature; a bubo ensued and was opened with the bistoury; the wound, however, soon degenerated into a deep ulcer, with hard edges and uneven unhealthy base. It extended to the scrotum and perineum, then to inner surface of the thigh, and continued, in spite of treatment, for six months. On the following summer, while the treatment consisted in tepid baths, fomentations, and the local application of lunar caustic, the ulcer suddenly took on the healing process and soon cicatrised.-Med. Jahrbücher, Nov., 1842.

\section{PRACTICAL OBSERVATIONS ON}

\section{DISEA SES OF THE SKIN.} By Thomas H. Burgess, M.D., \&c. No. III.

ERUPTIONS OF THE FACE.

Sycosis.-The history of sycosis or mentagra naturally follows that of acne, described in the preceding paper, inasmuch as it is a disease peculiar to the face, of an obstinate and rebellious character, and possesses many symptoms in common with that eruption. Indeed, some dermatologists seem to regard sycosis merely as a variety of acne rosacea (couperose) both being the result of a lesion of the follicles, and this view is in a manner supported by the circumstance of these diseases being pustular, and their anatomical seat apparently the same. For example, acne is produced by inflammation of the sebaceous follicles, and sycosis is pretty generally admitted to be the result of inflammation of the piliferous follicles; hence those who believe in the identity of the diseases in question say, that wherever one of these follicles is found the other is also present, and is equally involved. But this view of their identity is not correct, for sycosis is in reality the result of lesion of the piliferous tubes, in which the hair is encased as in a sheath, an opinion that has been fully substantiated by Biett and Cazenave. Moreover, these eruptions are further distinguished by the tubercular indurations of sycosis, showing that the subcutaneous cellular tissue is much involved, and especially by the abscence of hypersecretion of the sebaceous follicles, one of the principal phenomena of acne. In a word, sycosis is a pustular disease characterised by an eruption of accuminated pustules, although at a certain stage of its course, and under certain circumstances, it assumes a tubercular appearance.

Its seat is principally the chin, but it may appear wherever the beard grows, and it occurs most frequently in persons whose beard is thick and shaggy. The disease generally occurs in those parts of the face covered with hair, in the form of an eruption of small accuminated and painful pustules, especially on the chin, frequently on the upper lip, and sometimes along the maxillary region. Although, in some rare instance, this eruption runs its course pretty rapidly, and terminates in ten or fifteen days, it commonly assumes a chronic form-for example, a number of small pimples without any definite character, may 
appear and disappear repeatedly for several weeks, and even for months, until at length a true pustule makes its appearance. This generally brcaks about the third or fourth day, and is succeeded by a small scab, which in its turn falls off and leaves no traces behind. Another pustule now makes its appearance, and pursues the same course; and, finally, a number of pustules are evolved, accompanied by a painful sensation of tension, and redness sometimes strongly marked. The scabs increase in volume, become blackish, adherent, and when they do fall off are replaced by new pustules. The inflammation now extends to the adjoining tissues, slight tubercular engorgement ensues, and the disease assumes the appearance of a true tubercular affection. But we know that these tumors are not elementary, that they have succeeded the pustular eruption, that they are, in short, merely a secondary or consecutive lesion.

However, if the disease should still continue to pursue its course uninterupted, the tubercular indurations increase, and finally become true nodosities. The face is then greatly disfigured, the chin is puffy and swollen, and studded with tumors not unlike cherries in appearance, imparting to the countenance a peculiarly repulsive aspect. The inflammation may extend still more deeply into the cutaneous tissue, so as to produce abscesses, and then it is that we find sycosis complicated with the pustules of impetigo to such a degree as almost to disguise the original disease. When the eruption continues for some time, the bulbs participate in the surrounding inflammation, the hair is easily detached, sometimes falls off, some parts altogether, but it always grows again, and returns to its natural condition after a certain period.

The seat of sycosis, as already observed, may be the chin, maxillary region, eyelids, or upper lip. Sometimes it appears in the form of a single pustule on the nose, which goes through the usual course of the disease.

Its duration is very variable. It may resist every plan of treatment, and continue for an indefinite period in spite of the best directed remedial measures. The tubercular elevations will attain a considerable size if no treatment be employed; and especially, if the patient tears off the scabs at the summit, which he is likely to do from the itching of the parts, they will assume a livid color and extend over the chin, and even on to the cheeks. The tubcrcles are then so numerous and so large that the chin is more than double the natural size, and has an extremely repulsive appearance. When the disease shows a disposition to cure, the tubercles insensibly diminish in number and size; the scabs fall off, the pustules are less frequently renewed, and also diminish in number-in short, all the symptoms lose their intense character, and the eruption finally disappears. But red and violet colored patches frequently remain behind, especially when the duration of the disease was very prolonged, and slight epidermic exfoliation occurs in these parts.

Sycosis never attacks females, and very rarely old people, and this may be considered as a special character of the eruption. Those persons who have a thick and shaggy beard are much more predisposed to it than others. Some writers are of opinion that sycosis is a contagious affection, in consesequence of its having been transmitted from one person to another by using the same razor. M. Foville relates a case in support of this view, in which the disease attacked several of the patients in the lunatic asylum at Rouen from the use of a soiled razor. But it was an isolated example, and as the same occurrence did not take place again in the asylum under similar circumstances, it can only be looked upon as an accidental coincidence, and not as an argument in favor of the contagious nature of the disease under consideration.

There is evidently an individual predisposition in some persons to this eruption, which may be excited by occasional and accidental causes, as for example, the irritation produced by a blunt razor will suffice in such instances to produce sycosis. Amongst the exciting causes we may also mention the too frec use of intoxicating liquors, uncleanly habits, \&c. The seasons, also, seem to have considerable influence on the development of the disease, especially spring and autumn; and as a general rule it occurs most frequently in adults, especially those of a sanguineous and bilious temperament.

Sycosis is always easily distinguished from every other affection, unless it be complicated with the pustules of impetigo, in the event of which some attention and tact are necessary to discover the nature of the elementary disease. 'There are, however, certain diagnostic signs characteristic of each eruption, sufflcient to prevent their being confounded together. For example, the impetiginous pustules are not prominent, but flattened and partly incrusted together whenever those of sycosis are accuminated and distinct. The scabs of impetigo are broad, thick, and yellow, whilst the incrustations of sycosis are brownish and dry. When acne is developed on the chin it bears some resemblance to sycosis, but by bearing in mind that the pustules of the former are more superficial and inflammatory; that they do not suppurate completely; that the tubercular indurations are less pronounced; and finally, that the special character of acne-namely, hypersecretion of the follicles, is wanting - a mistake of this kind may be avoided.

In like manner, a knowledge of the general characters of sycosis, already enumerated, will prevent that disease being confounded with the tubercular or pustular syphilitic eruption. Sycosis is an obstinate affection and subject to frequent relapses; hence, it is necessary to be cantious in giving a prognosis, and especially in promising a speedy cure.

Treatment.-The treatment of this disease is often difficult and unsatisfactory, from the rebellious character it assumes, and sometimes it will baffle, for a considerable period, the best directed and most judicious remedial measures. In the acute stage, emollient cataplasms, employed every evening for a fortnight or three weeks, will be the most appropriate treatment that can be adopted. Cauterisation with the nitrate of silver is much recommended by $M$. Devergie in the tubercular form of the disease, together with the repeated employment of the vapor douche. A course of mild laxatives will also be serviceable as an adjuvant during the local treatment; and M. Biett was in the habit of employing emollient baths, containing a certain quantity of bran, starcl, or gelatine. M. Cazenare entertains an opposite opinion to that of 
M. Devergie regarding the utility of cauterisation, blisters, \&c., in the treatment of sycosis. M. Cazenave alleges that he never found any beneficial results to be derived from the application of these remedies.

When the disease assumes a chronic character, emollient applications are the most appropriate remedies to begin with; and after continuing these for a certain period, the duration of which will depend on the degree of severity of the disease, the best effects will often result from the judicious employment of the vapor douche. Mild laxatives, as the sulphates of potass or soda, should be occasionally administered during the treatment, and the administration of vegetable tonics or bitters will also tend to promote a curc. If this plan of treatment, however, should fail, we must then have recourse to certain topical remedies, the employment of which would not be judicious at any earlier period of the disease. These are ointments of tannin, of carbonate of lead; the sulphur ointment, so injurious in the acute forms of sycosis, may now be used with advantage; the iodine ointment, ointment of the proto-ioduret of mercury, or the red precipitate ointment. At the same time that these applications are used externally, the patient should take the sulphur waters internally and use the sulphur bath, if practicable. The ointments of the proto-ioduret of mercury and of the ioduret of sulphur should contain two grains of the salt to thirty grains of lard. If there is much local irritation present, the application of leeches behind the ears or under the chin will be attended with benefit; and if the employment of the local remedies above indicated produce a new eruption in greater abundance than ever, a circumstance which sometimes occurs, the treatment should be suspended, and the new symptoms attacked by the repeated applications of the vapor and alkaline douche.

London, Jan. 16, 1843.

\section{TWO CASES}

of

\section{COMPOUND FRACTURE OF THE LEG.}

By Jonathan Toogood, Esq., Senior Surgeon to the Bridgwater Infirmary.

CASE I.-Mr. A., aged fifty-five, was thrown from a gig on Thursday, and suffered a compound fracture of the leg. I found him lying on the road, about a mile from his house, bleeding profusely. He was carefully carried home on a hurdle, when I discovered that the lower end of the tibia was dislocated and considerably displaced, the fibula fractured, and about four inches above both bones were broken, the tibia protruding through a wound two inches in length. The tibia and fibula were again both broken a little below the knee. The reduction of the dislocation was very difficult, and it was impossible to keep the end of the tibia, which had passed through the skin, right, in consequence of the complete detachment of the bone by the upper fracture. The broken ends of the bones were placed in apposition, as well as the case would admit, the leg put up into splints, and cold applications directed. It became a point of serious consideration whether amputation would not be the safest course under such complicated injury. The patient was fifty-five years of age, and, with the exception of being subject to nervous and spasmodic affections, of a sound constitution and temperate habits; the soft parts, although much lacerated, were not contused, and he was in a situation possessing every convenience for cure. These circumstances induced me to hope that the first symptoms would not be such as to endanger his life, and that if the suppuration was not, excessive the limb might be preserved; I therefore felt justified in the attempt to save it.

Thursday night, Ten o'clock. He is restless and uneasy, complaining of great pain and starting of the limb, oppression and anxiety about the præcordia, with sickness and frequent tendency to evacuate the bowels. He took forty drops of laudanum, and afterwards an effervescing draught every four hours. The pulse 60 , with but little heat of skin. He got a little sleep during the night, and was much the same on the follow. ing morning, but complained of pain in making a deep inspiration; he still complained of tenesmus, and could not pass any water. The catheter was introduced with great relief. He continued in the same state during the day, the pulse varying from 60 to 68 ; but in the evening the oppression and languor was much increased, and the tongue became very dry. The wound looked pale and weak, and there was much discoloration of the foot, particularly of that part where the pressure necessary for the reduction of the dislocation had been made. The limb was well fomented; fifty drops of laudanum were given in some Madeira and water, and an injection administered, which was repeated at three in the morning, having been extremely restless, and without sleep. Immediate relief followed; he became comfortable and slept.

He passed the whole of the following day (Saturday) better; the same treatment was pursued, with the opium and wine; and he took chicken broth and beef tea.

Sunday. He complained of being very low, but all the other symptoms proceeded favorably.

On Monday the limb looked well, and there was a slight appearance of suppuration; his countenance and general state were improved, and he took some nutritive food with relish. The heat of this day was oppressive, the thermometer standing at 90 in the shade, and in the evening he was excessively low, and his voice so weak that he could scarcely be heard. A full dose of opium in Madeira procured a good night, and he passed the next day (Tuesday) well. Cordials and tonics were prescribed, and in the evening there was a sufficient discharge of healthy matter from the wound. The bladder recovered its power on the eighth day and everything went on favorably until the twelfth, excepting that no evacuations could be procured by glysters, but on that day the wound became dry. On the following evening, after passing the best day since the accident, violent spasms and shooting pains came on, affecting the leg, and particularly the muscles on the inside of the thigh. These spasmodic twitchings recurred frequently for many days, affecting the muscles of both legs, body, arms, and face. They generally came on as he was going to sleep, and somes awoke him from sound sleep. The granulations looked flabby; the wound was exquisitely tender, and presented a marbled puffy appearance. These symptoms excited some apprehension that tetanus may 